JOURNAL OF INTEGRAL EQUATIONS

AND APPLICATIONS

Volume 8, Number 1, Winter 1996

\title{
CONVERGENCE ESTIMATES FOR SOLUTION OF INTEGRAL EQUATIONS WITH GMRES
}

\author{
S.L. CAMPBELL, I.C.F. IPSEN, C.T. KELLEY, C.D. MEYER AND Z.Q. XUE
}

\begin{abstract}
In this paper we derive convergence estimates for the iterative solution of nonsymmetric linear systems by GMRES. We work in the context of strongly convergentcollectively compact sequences of approximations to linear compact fixed point problems. Our estimates are intended to explain the observations that the performance of GMRES is independent of the discretization if the resolution of the discretization is sufficiently good. Our bounds are independent of the righthand side of the equation, reflect the $r$-superlinear convergence of GMRES in the infinite dimensional setting, and also allow for more than one implementation of the discrete scalar product. Our results are motivated by quadrature rule approximation to second-kind Fredholm integral equations.
\end{abstract}

1. Introduction. In this paper we derive convergence estimates for the iterative solution of nonsymmetric linear systems by GMRES [25]. We work in the context of strongly convergent-collectively compact [2] sequences of approximations to linear compact fixed point problems. Our estimates are intended to explain the observations that the performance of GMRES is independent of the discretization if the resolution of the discretization is sufficiently good. Our bounds are independent of the righthand side of the equation, reflect the $r$-superlinear convergence of GMRES in the infinite dimensional setting, and also allow for more than one implementation of the discrete scalar product. This latter property is important in the context of integral equations, where an integral operator may be discretized with a high-order quadrature rule, with the implicit approximation of the $L^{2}$ inner product by that quadrature rule, and GMRES implemented in software that uses the

\footnotetext{
Received by the editors in revised form on October 24, 1995.

Key words and phrases. Integral equations, GMRES iteration, compact fixedpoint problem.

AMS (MOS) Subject Classifications. 65F10, 65J10, 65R20.

This research was supported by National Science Foundation grants DMS 9122745, CCR 9102853, CCR 9400921, DMS 9321938, DMS 9020915 and DMS 9403224. Computing activity was also partially supported by an allocation of time from the North Carolina Supercomputing Center.

Copyright (C)1996 Rocky Mountain Mathematics Consortium
} 
standard Euclidean inner product. We state our results in complex, rather than real, spaces because resolvent integration is an important component of our proofs.

Examples of sequences of strongly convergent-collectively compact maps arise in the approximate solution of linear second-kind Fredholm integral equations $[\mathbf{3}, \mathbf{2}, \mathbf{1 3}]$, elliptic partial differential equations which are preconditioned by solvers for high order terms $[\mathbf{9}, \mathbf{8}, \mathbf{2 0}, \mathbf{7}, \mathbf{6}]$, radiative transport problems $[\mathbf{2 4}, \mathbf{1 9}, \mathbf{1 5}]$, and semiconductor device modeling $[\mathbf{1 0}, \mathbf{1 1}, \mathbf{1 8}]$. In some of these papers, $[\mathbf{8}, \mathbf{1 5}, \mathbf{1 3}]$, GMRES is used as a coarse mesh solver in the context of a multilevel method and/or as an iterative solver for the linear equation for a Newton step in a nonlinear iteration. In other work $[\mathbf{7 , 6 , 1 8}]$, GMRES is used as a primary fine-mesh solver.

1.1. GMRES. This paper is concerned with convergence rate estimates, not implementation, and for our purposes it suffices to characterize the GMRES iteration in terms of the minimization property satisfied by the iterates [25]. In the setting of linear equations in a complex Hilbert space $H$,

$$
A u=f,
$$

the $l$ th GMRES iterate $u_{l}$ minimizes the $H$-norm of the residual $r_{l}=f-A u_{l}$ over vectors in the affine space $u_{0}+\mathcal{K}_{l}$, where $u_{0}$ is the initial iterate and the Krylov space $\mathcal{K}_{l}$ is given by

$$
\mathcal{K}_{l}=\operatorname{span}\left(r_{0}, A r_{0}, \ldots, A^{l-1} r_{0}\right) .
$$

We can express this minimization property as

$$
\left\|r_{l}\right\|_{H}=\min _{u \in u_{0}+\mathcal{K}_{l}}\|f-A u\|_{H} .
$$

If we let $\mathcal{P}_{l}$ be the class of residual polynomials [26],

$$
\mathcal{P}_{l}=\{p \mid p \text { is a polynomial of degree } l \text { and } p(0)=1\}
$$

it is easy to see, $[\mathbf{2 5}, \mathbf{1 4}]$, that

$$
r_{l}=f-A u_{l}=p_{l}(A) r_{0}
$$


for some $p_{l} \in \mathcal{P}_{l}$.

Equations (1.3) and (1.2) lead to the estimate

$$
\left\|r_{l}\right\|_{H}=\min _{p \in \mathcal{P}_{l}}|| p(A) r_{0}\left\|_{H} \leq\right\| r_{0}\left\|_{H} \min _{p \in \mathcal{P}_{l}}\right\| p(A) \mid \|_{\mathcal{L}(H)} .
$$

Here $\mathcal{L}(H)$ denotes the space of bounded operators on $H$ with the standard operator norm. Typical estimates for the convergence rate of GMRES select specific polynomials $p_{l} \in \mathcal{P}_{l}$ and then estimate the right side of (1.4) using

$$
\min _{p \in \mathcal{P}_{l}}\|p(A)\|_{\mathcal{L}(H)} \leq\left\|p_{l}(A)\right\|_{\mathcal{L}(H)} .
$$

Several papers have used (1.4), (1.5) and either diagonalization assumptions $[25,18,14,17]$, or resolvent integration methods $[22,23,4]$ to estimate $\left\|r_{l}\right\|_{H}$. We take the latter approach and seek to construct $p_{l}$ in such a way that families of operators can be handled with the same polynomial $p_{l}$. Our bounds reflect not only the superlinear convergence of GMRES for compact fixed point problems in Hilbert space [18, 23, $4]$ but also the manner in which the spectra of strongly convergent collectively compact families approximate that of the limiting operator. One can also, $[\mathbf{2 1}]$, obtain $q$-superlinear convergence results, but such bounds depend on the righthand side $f$.

1.2. Motivating example. When applied to discretizations of compact fixed point problems, integral equations in particular, more than one implementation of GMRES is possible. In this section we illustrate this with a simple example. This example will also serve to motivate our Banach-Hilbert space setting which, as was done in [9] in the context of Broyden's method, seeks to account for the successful application of an inner product (and hence Hilbert space) based algorithm to a problem naturally posed in a Banach space. A typical example of such a pairing of spaces is the solution of a problem posed in the Banach space of continuous functions on a compact set with GMRES and the $L^{2}$ scalar product.

Let $\Omega \subset R^{m}$ be compact, $k \in C(\Omega \times \Omega)$, and define the operator $K$ by

$$
K u(x)=\int_{\Omega} k(x, y) u(y) d y
$$


Clearly, $K \in \mathcal{C O M}\left(L^{2}(\Omega), C(\Omega)\right)$, the space of compact operators from $L^{2}$ to $C$. We set $A=I-K$ and seek to solve (1.1) with $f \in C(\Omega)$. We assume that $A$ is nonsingular. In the infinite dimensional setting, one could apply GMRES to (1.1) with $H=L^{2}(\Omega)$ and, [23, 4], expect $r$-superlinear convergence in the $L^{2}$ norm. One can also [17] obtain $r$-superlinear convergence in the $L^{\infty}$ norm by using an implicit form of Nyström interpolation.

We will approximate the operator $K$ by replacing the integral by a quadrature rule. We will consider a sequence of such approximations $\left\{K_{n}\right\}$, where

$$
K_{n}(u)(x)=\sum_{j=1}^{N_{n}} k\left(x, x_{j}^{n}\right) u\left(x_{j}^{n}\right) w_{j}^{n} .
$$

In (1.7) $\left\{x_{i}^{n}\right\}_{i=1}^{N_{n}}$ and $\left\{w_{i}^{n}\right\}_{i=1}^{N_{n}}$ are the nodes and weights of the quadrature rule used at level $n$. Throughout this paper we will assume that the weights of the quadrature rules are positive, that the quadrature rules integrate constant functions exactly, and that

$$
\lim _{n \rightarrow \infty} \sum_{j=1}^{N_{n}} g\left(x_{j}^{n}\right) w_{j}^{n}=\int_{\Omega} g(x) d x
$$

for all $g \in C(\Omega)$. For example, if $\Omega$ is an interval and the $n$th rule is a composite $p$-point Gauss rule on $n$ subintervals, $N_{n}=p n$.

It is well known [2], that the operators $K_{n}$ converge strongly to $K$ in $C(\Omega)$, i.e., $K_{n} u \rightarrow K u$ uniformly for all $u \in C(\Omega)$, and that the family $\left\{K_{n}\right\}$ is collectively compact, i.e., $\cup K_{n} B$ is precompact for any bounded set $B \subset C(\Omega)$. We set $A_{n}=I-K_{n}$. The solutions $u_{n}$ to

$$
A_{n} u=u-K_{n} u=f
$$

converge uniformly to $u^{*}=A^{-1} f$.

The solution $u_{n}$ of equation (1.8) may be solved [3] by first solving the finite dimensional fully discrete system

$$
u_{n}\left(x_{i}\right)-\sum_{j=1}^{N_{n}} k\left(x_{i}^{n}, x_{j}^{n}\right) u_{n}\left(x_{j}^{n}\right) w_{j}^{n}=f\left(x_{i}^{n}\right)
$$


for the values of $u_{n}$ at the nodal points of the quadrature rule and then recovering $u_{n} \in C(\Omega)$ by Nyström interpolation

$$
u_{n}(x)=f(x)+\sum_{j=1}^{N_{n}} k\left(x, x_{j}^{n}\right) u_{n}\left(x_{j}^{n}\right) w_{j}^{n} .
$$

At this point we have three approximations to $K . K_{n} \in \mathcal{L}(C(\Omega))$ is used in the analysis of the convergence of $u_{n}$ to $u^{*}$. The fully discrete approximation $K_{n}^{F D}: C^{N_{n}} \rightarrow C^{N_{n}}$ is defined for $V \in C^{N_{n}}$ by

$$
\left(K_{n}^{F D} V\right)_{i}=\sum_{j=1}^{N_{n}} k\left(x_{i}^{n}, x_{j}^{n}\right) V_{j} w_{j}^{n} .
$$

$K_{n}^{F D}$ is used in the finite dimensional system that one actually solves with GMRES. Finally the semidiscrete approximation $K_{n}^{S D}: C^{N_{n}} \rightarrow$ $C(\Omega)$ is defined for $V \in C^{N_{n}}$ by

$$
K_{n}^{S D} V(x)=\sum_{j=1}^{N_{n}} k\left(x, x_{j}^{n}\right) V_{j} w_{j}^{n} .
$$

The semidiscrete operator is used in Nyström interpolation (1.10).

The purpose of this paper is to derive bounds on the residuals of the GMRES iteration that apply to both the fully discrete (1.9) and continuous (1.1) problems. We will make an assumption on the structure of the sequence of operator approximations that will imply that the approximate operators have spectra that, in a sense sufficient for our purposes, approximate the spectrum of the limiting operator. This is not the case in general and problems with a high level of nonnormality can exhibit behavior far different from that of second kind Fredholm integral equations and their discretizations [5, 27].

Our work is related to the class of mesh independence results, first considered in $[\mathbf{1}]$ and $[\mathbf{1 6}]$ in the context of nonlinear problems. These results describe the convergence behavior of an iterative method, for example Newton's method, as it is applied to a sequence of approximations of an infinite dimensional problem. This was done in $[\mathbf{1}]$ and $[\mathbf{1 6}]$ by showing that the number of iterations required to drive the norm of 
the nonlinear residual to a given tolerance was independent of the level of approximation for sufficiently fine meshes. The name "mesh independence" was given to that phenomenon in [1]. Our notion of mesh independence for estimates of the GMRES residuals will be that the estimates are equally valid for the infinite dimensional problem (1.1) using the $L^{2}$ inner product and for the sequence of finite dimensional problems using an appropriate inner product on $C^{N_{n}}$. Moreover, these estimates are independent of the righthand side and allow for any natural choice of scalar product. We will describe the issue of choice of scalar product below.

We cannot apply GMRES directly to (1.8) in $L^{2}$ because the definition of $K_{n}$ depends on point evaluation. However, we can apply GMRES to the fully discrete problem (1.9) using either of two natural norms to solve the least squares problem for the GMRES iterate and compute the vector $U \in C^{N_{n}}$ where $U_{i}=u_{n}\left(x_{i}^{n}\right)$. We can put the standard Euclidean norm and scalar product on $C^{N_{n}}$, as would happen automatically if we used a general purpose code, or we could use the approximate $L^{2}$ norm and inner product that arise from the quadrature rule. In this latter case we would have

$$
(U, V)_{n}=\sum_{j=1}^{N_{n}} U_{j} \bar{V}_{j} w_{j}^{n}
$$

whereas in the former case

$$
(U, V)_{n}=\sum_{j=1}^{N_{n}} U_{j} \bar{V}_{j} .
$$

Multiplication of the inner product by a constant has no effect on the GMRES iteration, and we may scale the Euclidean inner product (1.14) by $m(\Omega) / N_{n}$, where $m$ is Lebesgue measure, to obtain

$$
(U, V)_{n}=\frac{m(\Omega)}{N_{n}} \sum_{j=1}^{N_{n}} U_{j} \bar{V}_{j} .
$$

The motivation for this scaling is that if $U$ is the vector with 1 in every component, then $(U, U)_{n}=m(\Omega)$ for all $n$ if $(\cdot, \cdot)_{n}$ is given by either (1.13) or (1.15). 
These different scalar products lead to different GMRES iterates as the least squares problems (1.2) are not the same. Our bounds are qualitatively the same for either choice of sequences of finite dimensional scalar products.

1.3. Main result. Our abstract setting is motivated by the example presented in Section 1.2. We formalize this setting in Assumption 1.1 in which care is taken to distinguish between the approximate problem (1.8), which is posed in the infinite dimensional space, the fully discrete (1.9), and the semidiscrete operator (1.12).

The convergence analysis in $[2]$ uses $\left\{K_{n}\right\}$ to show convergence of solutions of (1.8) to that of the infinite dimensional problem (1.1). The semidiscrete operators $\left\{K_{n}^{S D}\right\}$ are used in Nyström interpolation to connect the solution of the fully discrete problem to that of (1.8).

We will assume that $H, X, K,\left\{K_{n}\right\}$, and $\left\{K_{n}^{S D}\right\}$ satisfy

Assumption 1.1. 1. $H$ is a separable Hilbert space with scalar product $(\cdot, \cdot)$ and norm $\|\cdot\|_{H}$.

2. $X \subset H$ is a Banach space with norm $\|\cdot\|_{X}$.

3. $\|\cdot\|_{H} \leq\|\cdot\|_{X}$.

4. $K \in \mathcal{C O} \mathcal{M}(H, X), A=I-K$ is nonsingular.

5. $K_{n}$ converges strongly to $K$ in $X$, and the family $\left\{K_{n}\right\} \subset$ $\mathcal{C O} \mathcal{M}(X)$ is collectively compact.

6. There are numbers $N_{n}$, maps $P_{n}: X \rightarrow C^{N_{n}}$, and semidiscrete maps $K_{n}^{S D}: C^{N_{n}} \rightarrow X$ such that $P_{n}$ is onto and

$$
K_{n}=K_{n}^{S D} P_{n}
$$

7. There are a constant $C_{1}>0$ and scalar products $(\cdot, \cdot)_{n}$ defined on $C^{N_{n}}$ such that for all $n$, all $v \in X, V \in C^{N_{n}}$, and $\|\cdot\|_{n}=(\cdot, \cdot)_{n}^{1 / 2}$,

$$
\left\|P_{n} v\right\|_{n} \leq\|v\|_{X}
$$

and

$$
\left\|K_{n}^{S D} V\right\|_{X} \leq C_{1}\|V\|_{n}
$$


The role of the fully discrete operators is played by

$$
K_{n}^{F D}=P_{n} K_{n}^{S D}
$$

and

$$
A_{n}^{F D}=I-K_{n}^{F D} .
$$

The assumptions guarantee [2] that for $n$ sufficiently large $\left(I-K_{n}\right) u_{n}=$ $f$ may be uniquely solved for $u_{n}$ for any $f \in X$ and that $u_{n} \rightarrow u^{*}=$ $A^{-1} f$ in the norm of $X$.

To solve $u_{n}-K_{n} u_{n}=f$ we mimic the procedure described above in the integral equations case and first solve the fully discrete problem

$$
A_{n}^{F D} V=V-P_{n} K_{n}^{S D} V=P_{n} f
$$

for $V=P_{n} u_{n} \in C^{N_{n}}$. Then we recover $u_{n}$ through Nyström interpolation

$$
u_{n}=f+K_{n}^{S D} V .
$$

We will solve the finite dimensional system (1.20) with GMRES by solving the least squares problems for the GMRES iterates in the norm associated with the inner product $(\cdot, \cdot)_{n}$. Our assumptions imply that if $I-K_{n}$ is nonsingular on $X$, then $I-P_{n} K_{n}^{S D}$ is nonsingular on $C^{N_{n}}$. To see this, note that if $V-P_{n} K_{n}^{S D} V=0$ and $u=K_{n}^{S D} V$, then $P_{n} u=V$ and

$$
u-K_{n} u=u-K_{n}^{S D} P_{n} u=u-K_{n}^{S D} V=0,
$$

and therefore $u=0$ by nonsingularity of $I-K_{n}$. This implies that $V=0$ and hence $I-P_{n} K_{n}^{S D}$ is nonsingular.

In the context of the integral equation example above, Assumption 1.1 is satisfied if $H=L^{2}(\Omega),(\cdot, \cdot)$ is the $L^{2}(\Omega)$ inner product, $X=C(\Omega)$, and $X$ is given the scaled norm

$$
\|u\|_{X}=m(\Omega)^{1 / 2}|| u \|_{\infty},
$$

where $m$ denotes Lebesgue measure. $K$ and $K_{n}$ are defined by (1.6) and (1.7). If we define $K_{n}^{S D}$ by (1.12), then all parts of Assumption 1.1 with the possible exception of item 7 follow from the discussion in Section 1.2. 
Item 7 in Assumption 1.1 imposes a natural consistency condition between the inner product on $C^{N_{n}}$ used in GMRES and the discretization itself. Condition (1.16) requires that the vector in $C^{N_{n}}$ having 1 in each component be bounded as $n \rightarrow \infty$ in the norm

$$
\|\cdot\|_{n}=(\cdot, \cdot)_{n}^{1 / 2} .
$$

This holds for either of the scalar products (1.13) or (1.15) in Section 1.2 .

If $(\cdot, \cdot)_{n}$ is given by $(1.13)$, then since the weights of the quadrature rule are positive, it is clear that

$$
\left|K_{n}^{S D}(V)(x)\right| \leq\left.|| k\right|_{\infty} \sum_{j=1}^{N_{n}}\left|V_{j}\right| w_{j}^{n}
$$

for all $x \in \Omega$.

If we let $U \in C^{N_{n}}$ and $|V| \in C^{N_{n}}$ be the respective vectors having 1 and $\left|V_{j}\right|$ in each component, we have that

$$
\sum_{j=1}^{N_{n}}\left|V_{j}\right| w_{j}^{n}=(U,|V|)_{n} \leq\|U\|_{n}|| V\left\|_{n} \leq m(\Omega)^{1 / 2}\right\| V \|_{n} .
$$

We use (1.21) and (1.22) to obtain

$$
\left\|K_{n}^{S D} V\right\|_{X} \leq m(\Omega)\|k\|_{\infty}\|V\|_{n}
$$

and therefore (1.17) holds with $C_{1}=m(\Omega)\|k\|_{\infty}$.

If $(\cdot, \cdot)_{n}$ is the scaled Euclidean inner product given by $(1.15)$, we require a different bound on the right side of (1.22). In that case, if $W_{n} \in C^{N_{n}}$ has components $w_{j}^{n}$, then

$$
\sum_{j=1}^{N_{n}}\left|V_{j}\right| w_{j}^{n}=\frac{N_{n}}{m(\Omega)}\left(|V|, W_{n}\right)_{n}
$$

Hence

$$
\sum_{j=1}^{N_{n}}\left|V_{j}\right| w_{j}^{n} \leq \frac{N_{n}}{m(\Omega)}|| W_{n}\left\|_{n}\right\| V \|_{n}
$$


Hence, (1.17) will hold if $\left\{N_{n}\left\|W_{n}\right\|_{n}\right\}$ is bounded and we can use

$$
C_{1}=\|k\|_{\infty} m(\Omega)^{-1 / 2} \sup _{n}\left\{N_{n}\left\|W_{n}\right\|_{n}\right\}
$$

The right side of (1.23) is finite if and only if

$$
\left(N_{n}\left\|W_{n}\right\|_{n}\right)^{2}=N_{n} m(\Omega) \sum_{j=1}^{N_{n}}\left(w_{j}^{n}\right)^{2}
$$

is bounded. For composite $p$ point Gauss rules with $n$ subintervals, with $\Omega=[0,1]$, for example, $N_{n}=n p$ and $w_{j}^{n} \leq 1 / n$. In that case

$$
N_{n} \sum_{j=1}^{N_{n}}\left(w_{j}^{n}\right)^{2}=n p \sum_{j=1}^{N_{n}}\left(w_{j}^{n}\right)^{2} \leq \frac{n p}{n} \sum_{j=1}^{N_{n}} w_{j}^{n}=p
$$

for all $n$ and (1.17) holds.

We will prove our mesh independence results for GMRES using resolvent integration methods as was done in $[\mathbf{2 2}, \mathbf{2 3}]$ and $[4]$. The basic result, a perturbation insensitive form of an estimate in [4] relates convergence of GMRES for (1.1) to the spectrum and the algebraic multiplicity of eigenvalues of $A$ that are far in some sense from 1 . This result gives a sequence of residual polynomials $\left\{p_{l}\right\}$ that can be used to estimate not only the convergence rate of GMRES for (1.1) but also for (1.9).

Our main result is

Theorem 1.1. Let $H, X, K,\left\{K_{n}\right\}$, and $\left\{K_{n}^{S D}\right\}$ satisfy Assumption 1.1 , and let $\rho \in(0,1)$. Then there is $M_{1}(\rho)>0$, depending only on $\rho$ and $K$, such that if $n$ is sufficiently large and $r_{l}^{n}$ is the residual for the lth GMRES iteration for (1.20), then

$$
\left\|r_{l}^{n}\right\|_{n} \leq M_{1}(\rho) \rho^{l}\left\|r_{0}^{n}\right\|_{n}
$$

for all $l \geq 0$.

In Section 2 we prove Theorem 1.1. We begin with a discussion of some consequences of Assumption 1.1 that will allow us to make 
resolvent integral estimates that are independent of $n$. We then construct a family of residual polynomials and make the estimates. Our proof represents a simplification of the analysis in $[\mathbf{2 3}, \mathbf{1 8}]$ and $[4]$ and can be used without modification to prove that if $r_{l}$ is the GMRES residual for infinite dimensional iteration that

$$
\left\|r_{l}\right\|_{H} \leq M_{1}(\rho) \rho^{l}\left\|r_{0}\right\|_{H}
$$

for all $l \geq 0$. This implies $r$-superlinear convergence of the GMRES iteration when applied to linear compact fixed point problems in Hilbert spaces. If $K$ is diagonalizable (similar to a normal operator) or if the spectrum of $K$ satisfies certain growth properties, then superlinear rate estimates can be made directly in terms of the eigenvalues of $K$. Results of this kind can be found in $[\mathbf{1 7}, \mathbf{1 8}]$ and $[\mathbf{2 3}]$.

2. Proof of Theorem 1.1. This section is divided into two parts. In the first, Section 2.1, we prove some simple lemmas based on Assumption 1.1. Then, in Section 2.2, we apply those lemmas and resolvent integration methods to prove Theorem 1.1.

2.1. Consequences of Assumption 1.1. We begin our proof by deriving uniform estimates on the norms of inverses of fully discrete operators of the form

$$
z I-K_{n}^{F D}
$$

in $C^{N_{n}}$ with respect to the norm $\|\cdot\|_{n}$. Here $z \notin \sigma(K)$ the spectrum of $K$. These bounds will follow from similar $X$-norm bounds on $\left(z I-K_{n}\right)^{-1}$ that can be found in $[\mathbf{2}]$. We will also use the results from [2] on convergence of the spectra of the operators $K_{n}$ to obtain identical results for $K_{n}^{F D}$.

We will let $\|\cdot\|_{n}$ denote not only the norm on $C^{N_{n}}$ induced by the scalar product $(\cdot, \cdot)_{n}$ but also the induced matrix norm.

Lemma 2.1. Let Assumption 1.1 hold, and let $\mathcal{S}$ be a compact set in the complex plane that is disjoint from the spectrum of $K$. Then there is $C_{2}(\mathcal{S})$ so that, for $n$ sufficiently large, $z I-K_{n}^{F D}$ is nonsingular on $C^{N_{n}}$ and

$$
\left\|\left(z I-K_{n}^{F D}\right)^{-1}\right\|_{n} \leq C_{2}(\mathcal{S})
$$


for all $z \in \mathcal{S}$.

Proof. Assumption 1.1 implies that for $n$ sufficiently large $\left(z I-K_{n}\right)$ is nonsingular for all $z \in \mathcal{S}$, and there is an $M$ such that

$$
\left\|\left(z I-K_{n}\right)^{-1}\right\|_{\mathcal{L}(X)} \leq M
$$

for all $z \in \mathcal{S}$. As we pointed out in Section 1.3, nonsingularity of $z I-K_{n}$ implies that of $z I-K_{n}^{F D}$. Hence it remains only to prove the bound (2.1).

Let $G \in C^{N_{n}}$, and let $g \in X$ be such that $G=P_{n} g$. Such a $g$ exists by our assumption that $P_{n}$ is an onto map. Let $V=\left(z I-K_{n}^{F D}\right)^{-1} G$, and let $v=z^{-1}\left(g+K_{n}^{S D} V\right)$. Note that $v$ is well defined because $0 \in \sigma(K)$ and therefore not in $\mathcal{S}$. We have $P_{n} v=V$ and

$$
\begin{aligned}
z v & =g+K_{n} v=g+K_{n}\left(z I-K_{n}\right)^{-1} g \\
& =g+\left(z I-K_{n}\right)^{-1} K_{n} g=g+\left(z I-K_{n}\right)^{-1} K_{n}^{S D} G .
\end{aligned}
$$

Hence,

$$
z V=z P_{n} v=G+P_{n}\left(z I-K_{n}\right)^{-1} K_{n}^{S D} G
$$

and therefore, using Assumption 1.1, we have

$$
\|V\|_{n} \leq|z|^{-1}\left(1+M C_{1}\right)\|G\|_{n} .
$$

If we set

$$
C_{2}(\mathcal{S})=\left(1+M C_{1}\right) \max _{z \in \mathcal{S}}|z|^{-1}
$$

the proof is complete.

The next lemma expresses convergence of the spectrum in a way that we can use directly in our analysis. Let $L$ be a compact operator, $B=I-L$, and $\rho \in(0,1)$ be given. Let $\left\{\lambda_{j}\right\}_{j=1}^{m}$ be the eigenvalues of $B$ satisfying

$$
\left|1-\lambda_{j}\right|>\rho,
$$

and counted by multiplicity. We define a residual polynomial

$$
p(z, L, \rho)=\prod_{j=1}^{m}\left(1-z / \lambda_{j}\right) .
$$


Note that the definition of $p(z, L, \rho)$ has nothing to do with the particular space on which $L$ is defined. The requirement that $L$ be compact is sufficient to guarantee that the product in (2.3) is finite for any $\rho>0$. It is possible for $m$ to vanish, for instance, if $\rho>\|L\|$ or $L$ is quasinilpotent. In that event the product in (2.3) is empty, and $p=1$, as is standard. $p$ is the characteristic polynomial for the eigenvalues of $B$ outside the disk of radius $\rho$ about $z=1$. In [4] the analogous minimal polynomial was used. We must use the characteristic polynomial here because we analyze a sequence of operators.

Lemma 2.2 follows from the results in [2].

Lemma 2.2. Let Assumption 1.1 hold, and let $\rho \in(0,1)$ be given. Then

$$
\lim _{n \rightarrow \infty} p\left(z, K_{n}^{F D}, \rho\right)=p(z, K, \rho)
$$

uniformly on compact sets in the complex plane.

2.2. Completion of the proof. Let $\rho \in(0,1)$ be given, and let $C_{\rho}$ denote the circle of radius $\rho$ about $z=1$ in the complex plane. If necessary, we reduce $\rho$ so that no eigenvalue of $A$ lies on $C_{\rho}$.

Let $\left\{\lambda_{j}\right\}_{j=1}^{m}$ be the eigenvalues of $A$ which lie outside $C_{\rho}$, i.e., the roots of $p(z, K, \rho)$. Lemma 2.2 implies that, for $n$ sufficiently large, there are exactly $m$ eigenvalues (counted by multiplicity) of $A_{n}^{F D}=I-K_{n}^{F D}$ outside $C_{\rho}$. Following [4], we separate the spectrum into a "good" part inside $C_{\rho}$ and a "bad" part outside. Our estimate will be based on construction of a residual polynomial that has roots at the eigenvalues of $A_{n}^{F D}$ that are outside $C_{\rho}$ and then provides an $r$-linear convergence estimate with $r$-factor at most $\rho$. The fact that if $K$ is compact then any $\rho \in(0,1)$ can be used in such a decomposition of $\sigma(A)$ implies $r$-superlinear convergence [4].

Let $n$ be large enough so that the number of eigenvalues of $A$ and $A_{n}^{F D}$ outside $C_{\rho}$ is the same and is $m$. Let

$$
\alpha_{n}(\rho)=\max _{z \in C_{\rho}}\left|p(z, K, \rho)-p\left(z, K_{n}^{F D}, \rho\right)\right|,
$$

and increase $n$ if needed so that

$$
\alpha_{n}(\rho) \leq 2 \max _{z \in C_{\rho}}|p(z, K, \rho)| .
$$


For $l \geq m$ we define residual polynomials $p_{l} \in \mathcal{P}_{l}$ by

$$
p_{l}(z)=(1-z)^{l-m} p\left(z, K_{n}^{F D}, \rho\right) .
$$

We define spectral projections

$$
\Pi_{G}=\frac{1}{2 \pi i} \int_{C_{\rho}}\left(z I-A_{n}^{F D}\right)^{-1} d z \quad \text { and } \quad \Pi_{B}=I-\Pi_{G} .
$$

We use (1.5), the polynomial $p_{l}$, and the spectral projections defined in (2.5) to obtain (see [12] and [4])

$$
\begin{aligned}
p_{l}\left(A_{n}^{F D}\right) & =\Pi_{G} p_{l}\left(A_{n}^{F D}\right)+\Pi_{B} p_{l}\left(A_{n}^{F D}\right) \\
& =\Pi_{G} p_{l}\left(A_{n}^{F D}\right) \\
& =\frac{1}{2 \pi i} \int_{C_{\rho}}\left(z I-A_{n}^{F D}\right)^{-1} p_{l}(z) d z
\end{aligned}
$$

and hence

$$
\left\|p_{l}\left(A_{n}^{F D}\right)\right\|_{n} \leq D_{1} \rho^{l}
$$

where, letting $D_{\rho}=\{|z|=\rho\}$,

$$
D_{1}=\rho^{1-m} C_{2}\left(D_{\rho}\right) \max _{z \in C_{\rho}}\left|p\left(z, K_{n}^{F D}, \rho\right)\right| .
$$

In view of (1.4) and (2.6), we may complete the proof by noting that

$$
\left|p\left(z, K_{n}^{F D}, \rho\right)\right| \leq|p(z, K, \rho)|+\alpha_{n}(\rho) \leq 3 \max _{z \in C_{\rho}}|p(z, K, \rho)|
$$

and setting

$$
M_{1}(\rho)=2 \rho^{1-m} C_{2}\left(D_{\rho}\right) \max _{z \in C_{\rho}}|p(z, K, \rho)| .
$$

\section{REFERENCES}

1. E.L. Allgower, K. Böhmer, F.A. Potra and W.C. Rheinboldt, A meshindependence principle for operator equations and their discretizations, SIAM J. Numer. Anal. 23 (1986), 160-169. 
2. P.M. Anselone, Collectively compact operator approximation theory, PrenticeHall, Englewood Cliffs, New Jersey, 1971.

3. K.E. Atkinson, A survey of numerical methods for Fredholm integral equations of the second kind, SIAM, Philadelphia, 1976.

4. S.L. Campbell, I.C.F. Ipsen, C.T. Kelley and C.D. Meyer, GMRES and the minimal polynomial, Tech. Report. CRSC-TR94-10, Center for Research in Scientific Computation, North Carolina State University, July 1994.

5. F. Chaitin-Chatelin, Is nonnormality a serious difficulty?, Tech. Report. TR/PA/94/18, CERFACS, December 1994.

6. S.C. Eisenstat and H.F. Walker, Choosing the forcing terms in an inexact Newton method, Tech. Report 6/94/75, Mathematics and Statistics Department, Utah State University, June 1994.

7. Globally convergent inexact Newton methods, SIAM J. Control Optim. 4 (1994), 393-422.

8. M. Heinkenschloss, C.T. Kelley and H.T. Tran, Fast algorithms for nonsmooth compact fixed point problems, SIAM J. Numer. Anal. 29 (1992), 1769-1792.

9. D.M. Hwang and C.T. Kelley, Convergence of Broyden's method in Banach spaces, SIAM J. Control Optim. 2 (1992), 505-532.

10. J.W. Jerome, Consistency of semiconductor modeling: An existence/stability analysis for the stationary von Roosbroeck system, SIAM J. Appl. Math. 45 (1985), $565-590$.

11. - The role of semiconductor device diameter and energy-band bending in the convergence of Picard iteration for Gummel's map, IEEE Trans. Elec. Dev 32 (1985), 2045-2051.

12. T. Kato, A short introduction to perturbation theory for linear operators, Springer Verlag, New York, 1982.

13. C.T. Kelley, A fast multilevel algorithm for integral equations, SIAM J. Numer. Anal. 32 (1995), 501-513.

14. - Iterative methods for linear and nonlinear equations, SIAM Frontiers Appl. Math. 16, SIAM, Philadelphia, 1995.

15. - Multilevel source iteration accelerators for the linear transport equation in slab geometry, Trans. Theory Statist. Phys. 24 (1995), 679-708.

16. C.T. Kelley and E.W. Sachs, Broyden's method for approximate solution of nonlinear integral equations, J. Integral Equations 9 (1985), 25-44.

17. C.T. Kelley and Z.Q. Xue, GMRES and integral operators, Tech. Report CRSC-TR94-7, North Carolina State University, Center for Research in Scientific Computation, April 1994.

18. T. Kerkhoven and Y. Saad, On acceleration methods for coupled nonlinear elliptic systems, Numer. Math. 60 (1992), 525-548.

19. E.W. Larsen and P. Nelson, Finite difference approximations and superconvergence for the discrete ordinate equations in slab geometry, SIAM J. Numer. Anal. 19 (1982), 334-348.

20. T.A. Manteuffel and S. Parter, Preconditioning and boundary conditions, SIAM J. Numer. Anal. 27 (1990), 656-694. 
21. I. Moret, A note on the superlinear convergence of GMRES, preprint.

22. N.M. Nachtigal, S.C. Reddy and L.N. Trefethen, How fast are nonsymmetric matrix iterations?, SIAM J. Matrix Anal. Appl. 13 (1992), 778-795.

23. O. Nevanlinna, Convergence of iterations for linear equations, Birkhäuser, Basel, 1993.

24. J. Pitkäranta and R. Scott, Error estimates for the combined spatial and angular approximations of the transport equation in slab geometry, SIAM J. Numer. Anal. 20 (1983), 922-950.

25. Y. Saad and M. Schultz, GMRES a generalized minimal residual algorithm for solving nonsymmetric linear systems, SIAM J. Sci. Statist. Comput. 7 (1986), 856-869.

26. E.L. Stiefel, Kernel polynomials in linear algebra and their numerical applications, U.S. National Bureau of Standards, Applied Mathematics Series 49 (1958), $1-22$.

27. L.N. Trefethen, A.E. Trefethen, S.C. Reddy and T.A. Driscoll, Hydrodynamic stability without eigenvalues, Science 261 (1993), 578-584.

Center for Research in Scientific Computation and Department of Mathematics, North Carolina State University, Box 8205, Raleigh, NC 27695-8205

E-mail: slc@math.ncsu.edu

Center for Research in Scientific Computation and Department of Mathematics, North Carolina State University, Box 8205, Raleigh, NC 27695-8205

E-mail: ipsen@math.ncsu.edu

Center for Research in Scientific Computation and Department of Mathematics, North Carolina State University, Box 8205, Raleigh, NC 27695-8205

E-mail: meyer@math.ncsu.edu

Center for Research in Scientific Computation and Department of Mathematics, North Carolina State University, Box 8205, Raleigh, NC 27695-8205

E-mail: Tim_Kelley@ncsu-edu

Center for Research in Scientific Computation and Department of Mathematics, North Carolina State University, Box 8205, Raleigh, NC 27695-8205

E-mail: xue@stokes.herl.epa.gov

Current address for Z.Q. Xue: Hughes Information Technology, 1616 McCormick Drive, Landover, MD 20785

E-mail: zxue@eos.hitc.com 